\title{
Flexible ureteroscopic renal stone extraction during laparoscopic ureterolithotomy in patients with large upper ureteral stone and small renal stones
}

\author{
Jae Hyung You, MD; Young Gon Kim, MD; Myung Ki Kim, MD
}

Department of Urology, Chonbuk National University Medical School and Research Institute of Clinical Medicine of Chonbuk National University-Biomedical Research Institute of Chonbuk National University Hospital, Jeonju, South Korea

Cite as: Can Urol Assoc J 2014;8(9-10):e591-4. http://dx.doi.org/10.5489/cuaj.1806 Published online September 9, 2014.

\section{Abstract}

Introduction: We describe laparoscopic ureterolithotomy with renal stone extraction using a stone basket under flexible ureteroscopy. We describe its efficacy through a laparoscopic port and a ureterotomy site in patients with large upper ureteral stone and small renal stones.

Methods: Between January 2009 and February 2012, we performed laparoscopic ureterolithotomy with renal stone extraction using a stone basket under flexible ureteroscopy in 11 patients who had upper ureteral and renal stones. The retroperitoneal approaches were used in all patients using 3-4 trocars.

Results: All procedures were performed successfully without significant complications. Mean operative time was 78.5 minutes (range: 52-114 minutes). The mean size of ureteral stone was $19.91 \mathrm{~mm}$ (range: $15-25 \mathrm{~mm}$ ). In addition, 25 renal stones (mean size 7.48 $\mathrm{mm}$, range: $2-12 \mathrm{~mm}$ ) were removed from 11 patients. The mean length of hospital stay was 3.5 days (range: 2-6 days).

Conclusions: Laparoscopic ureterolithotomy with renal stone extraction using a stone basket under flexible ureteroscopy can be considered one of treatment modalities for patients with large upper ureteral stones accompanied by renal stones who are indicated in laparoscopic ureterolithotomy.

\section{Introduction}

Current standard treatment modalities for upper and middle ureteral stones include extracorporeal shock wave lithotripsy (SWL), ureteroscopy and percutaneous antegrade removal. ${ }^{1-3}$

For patients who failed on these treatments, open surgery is needed. These patients include those with hard, large and impacted stones or who underwent minimally invasive techniques. ${ }^{4}$ Open surgery, however, has its disadvantages in that it increases the morbidity of patients. In most circumstances, a laparoscopic approach is a minimally invasive alternative to the open surgery.
There are some patients with upper ureteral stones for whom laparoscopic ureterolithotomy should be performed concomitantly with small renal stones. In these patients, renal stones should be treated with the additional use of SWL after operation. With the recent advances in the development of smaller calibre, flexible ureteroscopy lithotripter (URS) and intracorporeal lithotripter, the success rate in treating renal stones has greatly increased. Still, there are many limitations in the retrograde intra-renal surgery (RIRS). The retrograde flexible ureteroscopic renal stone removal through a laparoscopic port and a ureterotomy site is feasible compared with the RIRS and it is more effective than the postoperative SWL.

We report our experience of laparoscopic retroperitoneal ureterolithotomy with renal stone extraction using a stone basket under flexible ureteroscopy through a laparoscopic port and a ureterotomy site in patients with both large upper ureteral stone and small renal stones.

\section{Methods}

Between January 2009 and February 2012, we performed retroperitoneoscopic ureterolithotomy in 40 patients who had either large or impacted stones in the upper ureter and in whom SWL failed. Of these patients, 11 with large upper ureteral stone and renal stones underwent laparoscopic retroperitoneal ureterolithotomy with renal stone extraction using stone basket under flexible ureteroscopy through a laparoscopic port and a ureterotomy site.

The retroperitoneal approaches with lateral position were used in all patients. Patients were placed in a full lateral flank position, for which the table was elevated to flatten the lumbar region. Three laparoscopic trocars were placed in 7 and 4 trocars in 4 patients, 2 of whom had a high body mass index and the other 2 with a stone near the ureteropelvic junction. A 1.5-cm incision was made just below the tip of the 12 th rib. With the incision of the transversalis fascia, the 


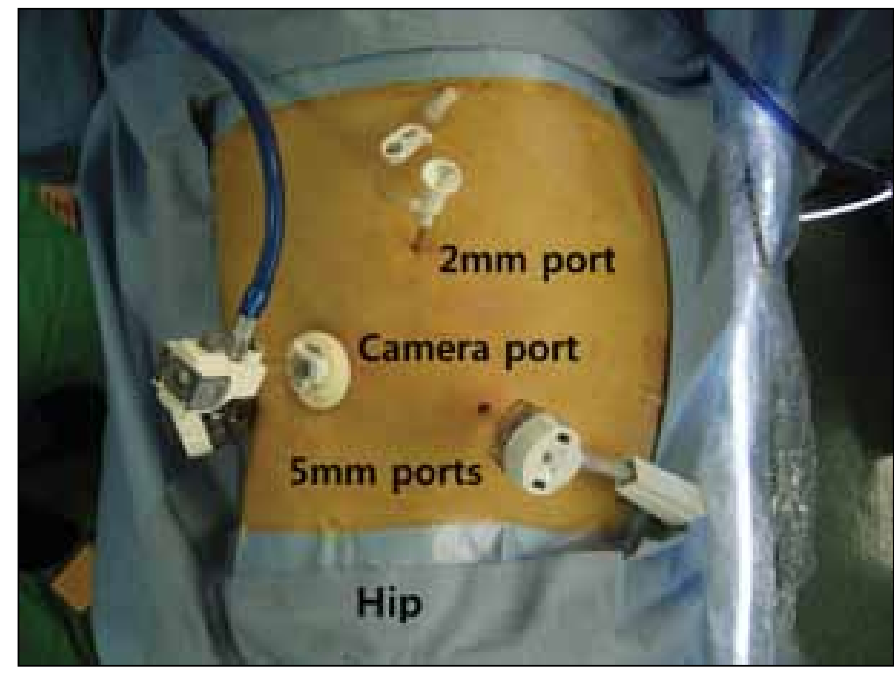

Fig. 1. The configuration of ports in the right ureteral and renal stones.

posterior pararenal space was bluntly created as remotely as possible with an index finger pushing the peritoneum forward. Moreover, the sufficient space was created to introduce the balloon dilator. We used a balloon dilator to create the retroperitoneal space and left the inflated balloon for a few minutes to achieve a hemostasis. A 10-mm balloon trocar was used as a camera port. A pneumoretroperitoneum was created at a pressure of $15 \mathrm{mmHg}$. A 5-mm trocar was introduced under endoscopic control 2 finger-breadths above the iliac crest on the anterior axillary line. This port served mainly for hand instrumentation throughout the procedure. The third 5-mm port was inserted under vision at the junction of the lateral border of paraspinal muscles and the 12th rib. Occasionally, if the patient was obese or had a stone near the ureteropelvic junction, the fourth 2-mm trocar was used for retraction (Fig. 1).

Usually the calculus was large enough to be identified, appearing as a ureteral bulge, and it was dissected with a blunt dissector or forceps through a port and a hook via the other port. The periureteral tissue was further cleared before making an incision. The ureterotomy was performed with a laparoscopic knife. The stone was removed with a grasping forceps or right angle forceps and then extracted.

After the ureteral stone removal, the flexible ureteroscopy was inserted into the ureter, renal pelvis and calyces through a laparoscopic port and a ureterotomy site. To identify the renal stones, the stone removal was done efficiently using a stone basket (Fig. 2a, Fig. 2b).

Because the upper portion of ureter was dilated from the ureteral stone, flexible ureteroscopy can get into the ureter without use of guide wires or ureteral catheter and renal stones were removed without fragmentation in all patients (Fig. 3a, Fig. 3b).

The Double J-stent was used formerly in 6 patients; it was indwelled by antegrade introduction of a $6 \mathrm{Fr} 26-\mathrm{cm}$ Double J-stent through the 2- or 5-mm trocar and the ureterotomy. The Double J-stent was passed down the ureter into the bladder first, then up the ureter to the renal pelvis. The ureterotomy site was closed with interrupted intracorporeal sutures using a 5-0 Vicryl. A 5-mm laparoscope was then passed through a 5-mm port to locate the stone and a 10-mm Babcock forceps were passed through the primary port site for the removal of intact stones. The stone in laparoscopic Babcock forceps was pulled out with the port. The primary port was reinserted and to ensure the final hemostasis and to aspirate any free fluid in the retroperitoneum. A tube drain was placed in the retroperitoneal space through a $5-\mathrm{mm}$ port site.

\section{Results}

A total of 11 patients underwent laparoscopic ureterolithotomy with a renal stone extraction using a stone basket under flexible ureteroscopy. The mean patient age was 58.0 years (range: $31-81$ ) and the male-to-female ratio was 8:3. All ureteral stones were located in the upper ureter.

\begin{tabular}{|c|c|c|c|c|c|c|c|}
\hline Number & Age/sex & Location & Stone size (mm) & Renal stone size (mm) & Operative time (min) & Ureter stent & Complications \\
\hline 1 & 71/M & Right upper & 25 & $5,5,5,5,10$ & 72 & + & - \\
\hline 2 & $57 / M$ & Left upper & 23 & 6,10 & 57 & + & - \\
\hline 3 & $41 / \mathrm{M}$ & Left upper & 15 & $2,3,4$ & 56 & + & - \\
\hline 4 & $63 / \mathrm{M}$ & Right upper & 15 & 7,11 & 114 & + & - \\
\hline 5 & $81 / F$ & Right upper & 25 & 7,11 & 80 & - & - \\
\hline 6 & $55 / \mathrm{M}$ & Right upper & 16 & 3,4 & 70 & + & $\begin{array}{c}\text { Urine leak } \\
\text { (spontaneously } \\
\text { corrected at } \\
\text { postoperative } 4 \text { days) }\end{array}$ \\
\hline 7 & $42 / \mathrm{M}$ & Left upper & 24 & 12 & 90 & - & - \\
\hline 8 & $31 / \mathrm{M}$ & Left upper & 17 & 10 & 52 & - & - \\
\hline 9 & 79/M & Left upper & 18 & $5,5,5,5$ & 70 & + & - \\
\hline 10 & $59 / F$ & Right upper & 21 & 5,7 & 98 & - & - \\
\hline 11 & $58 / \mathrm{F}$ & Right upper & 20 & 10 & 105 & - & - \\
\hline
\end{tabular}




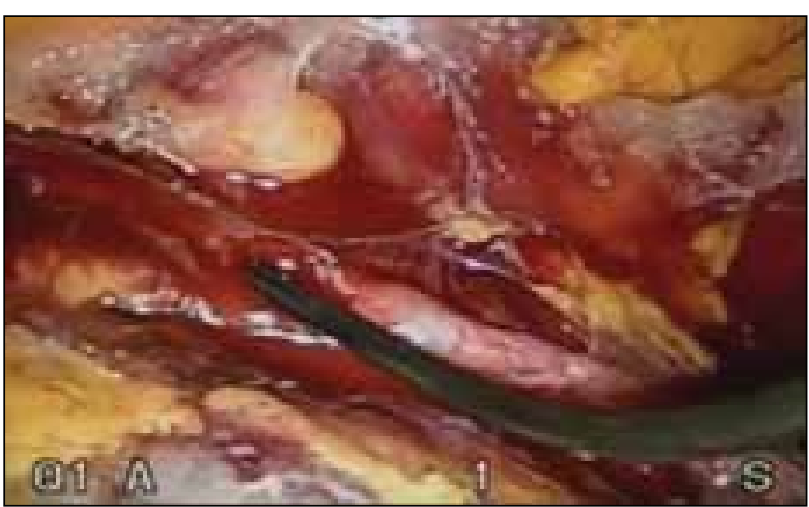

Fig. 2a. The operative findings of the laparoscopic ureterolithotomy with the removal of renal stones under flexible ureteroscopy.

There were no cases for which an open conversion was needed. The procedure could be completed using only 3 ports in 7 patients. But the fourth port was required in 4 patients. The mean operative time was 78.5 minutes (range: 52-114). The mean size of ureteral stone was $19.91 \mathrm{~mm}$ (range: 15-25). In addition, 25 renal stones (mean size $7.48 \mathrm{~mm}$ [range: 2-12]) were removed from 11 patients. The ureter was closed with an intracorporeal laparoscopic suture for which a stent was placed in 6 patients and no stent placement was done in 5 patients. The drain was removed after 1 to 2 days according to the status of drainage. There was 1 patient who had a urine leakage. In this patient, however, the leakage was spontaneously discontinued without any consequences on postoperative day 4 . The mean length of hospital stay was 3.5 days (range: 2-6). Every patient recovered without developing other notable complications. The mean follow-up length was 26.5 months and there were no complications, including ureteral stricture.

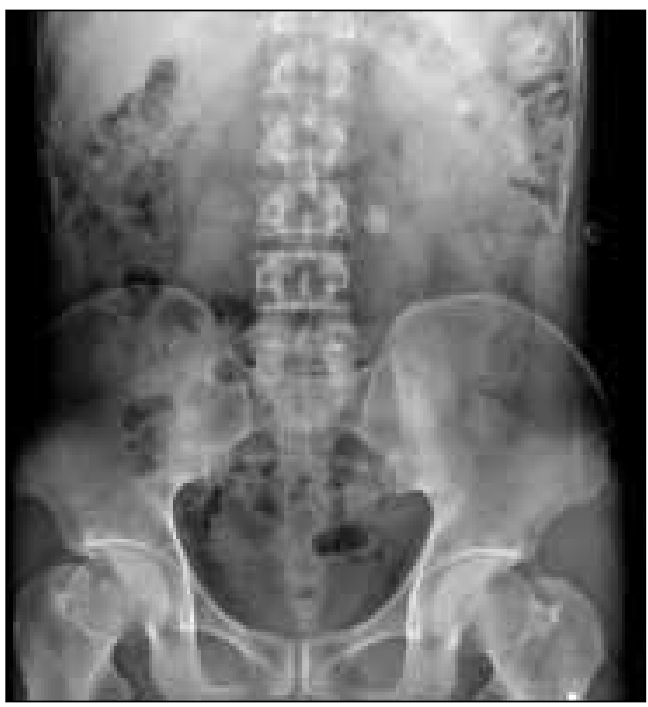

Fig. 3a. Preoperative findings of kidney-ureter-bladder that is suggestive of large upper ureteral stones with renal stones.

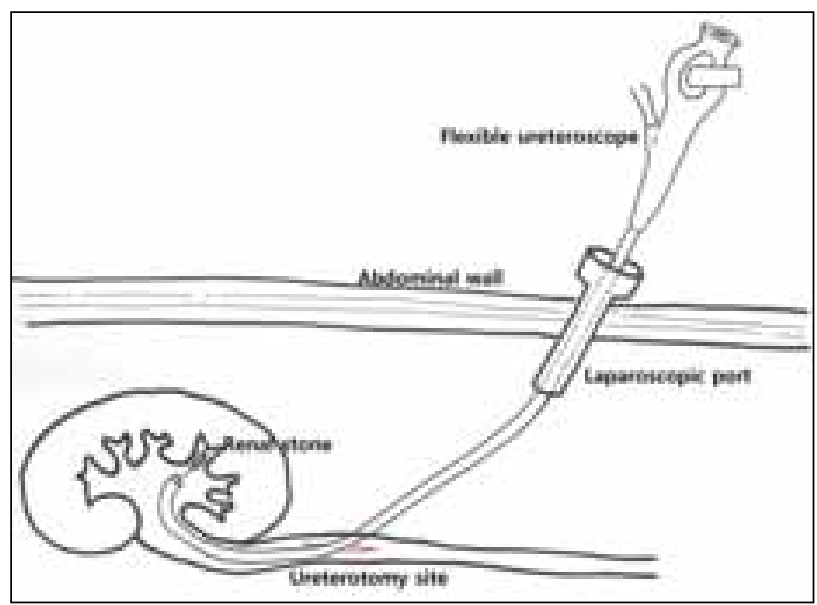

Fig. $\mathbf{2 b}$. Schematic illustration of the procedure.

\section{Discussion}

Ureteral stones frequently cause renal colic, which may lead to obstructive uropathy. Minimally invasive surgery is the preferred treatment modality for ureteral stones. The SWL and ureteroscopy are the most common interventions to remove the ureteral stones. ${ }^{5,6}$ However, if the ureteral stones are relatively larger and refractory to minimally invasive techniques, one can consider an open ureterolithotomy. A ureterolithotomy is a minimally invasive modality that can be done under the laparoscopic guidance; its success rate is similar to open surgery and its morbidity is relatively lower compared with open surgery. In 1979, Wickham first introduced the concept of retroperitoneal ureterolithotomy. ${ }^{7}$ Thereafter, Raboy and colleagues first performed a transperitoneal laparoscopic ureterolithotomy in $1992 .{ }^{8}$

Currently, laparoscopic ureterolithotomy is done either transperitoneally or retroperitoneally. Compared with a transperitoneal approach, a retroperitoneal approach is advantageous in that it requires no bowel mobilization, causes less injury to the colon or ileus and can be done both promptly and directly. Moreover, it can prevent the

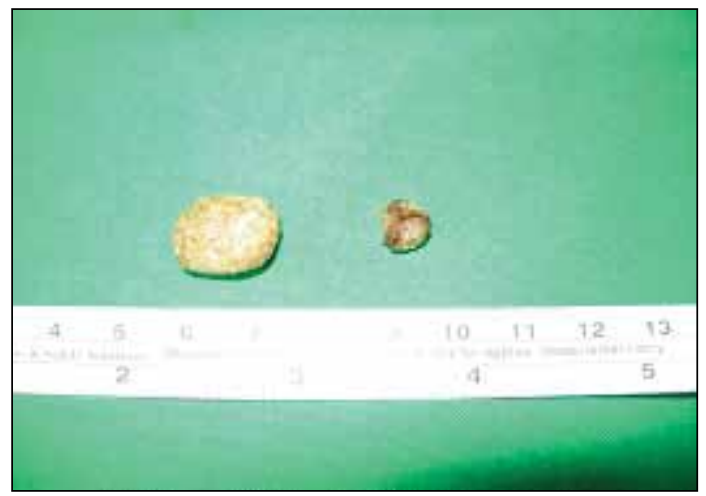

Fig. $\mathbf{3 b}$. Findings after the removal of the ureteral and renal stones. 
You et al.

contamination of the peritoneal cavity, causes no shoulder tip pain to patients, can be done regardless of the patient's medical history of abdominal surgery and has a low incidence of long-term complications, such as port site hernia and bowel obstruction. ${ }^{8-11}$ This is why we prefer a retroperitoneal approach.

We formerly managed some patients with upper or midureteral stones with renal stones although they were not indicated for surgical procedures, such as percutaneous nephrolithotomy (PCNL). Following the SWL, URS or laparoscopic ureterolithotomy for the removal of ureteral stone, we additionally performed the SWL for the treatment of renal stones in most of the patients.

There have been great advancements in SWL. Its success depends on the size and density of stone and the degree of ureteral obstruction. The rate of treatment success is $49 \%$ to $96 \% .^{12,13}$ Moreover, the additional treatments were needed in $43 \%$ of patients who underwent SWL because the ureteral obstruction occurred as a result of the movement of smashed stones. It is also known that harder stones are refractory to the treatment. ${ }^{12,13}$ Therefore, if possible, the renal stone should be removed synchronously with the ureteral stone.

With the advancements in flexible ureteroscope, instruments and user experience, RIRS has been increasingly used over the past decade. The current ureteroscopic management of renal calculi provides an alternative to SWL or PCNL. This could achieve higher stone-free rates than SWL with a lower morbidity than PCNL. ${ }^{14}$ But the ureteroscopic management of renal calculi reveals the limitation in the efficacy. Moreover, it does not ensure that patients would be devoid of renal stones. ${ }^{15}$ From our experiences, RIRS through a laparoscopic port and a ureterotomy site is a safe, feasible modality compared with conventional RIRS.

Sun and colleagues have recently described the methods for treating ipsilateral renal ureteral calculi by combining retroperitoneal laparoscopic surgery with tubeless minipercutaneous nephrolithotomy. ${ }^{16}$

In our experience, laparoscopic ureterolithotomy with renal stone extraction using a stone basket under flexible ureteroscopy shortens the postoperative course and accelerates a recovery. It may even play a key role in the treatment of urinary stones.

\section{Conclusion}

Laparoscopic ureterolithotomy with renal stone extraction using a stone basket under flexible ureteroscopy is a safe, feasible modality. It can be considered a treatment modality for patients with large upper ureteral stones accompanied by renal stones that are indicated in laparoscopic ureterolithotomy.

Acknowledgments: This paper was supported by Fund of Biomedical Research Institute, Chonbuk National University Hospital.

Competing interests: Dr. You, Dr. Y.G. Kim and Dr. M.K. Kim all declare no competing financial or personal interests.

This paper has been peer-reviewed.

\section{References}

1. Drach GW, Dretter S, Fair W, et al. Report of the United States Cooperative Study of Extracorporeal Shock Wave Lithotripsy. J Urol 1986;135:1127.

2. Liong ML, Clayman RV, Gittes RF et al. Treatment options for proximal ureteral urolithiasis: Review and recommendations. J Urol 1989; 141:504-9.

3. Blute ML, Segura JW, Patterson DE. Ureteroscopy. J Urol 1988;139:510-2.

4. Gaur DD, Trivedi S, Prabhudesai MR, et al. Laparoscopic ureterolithotomy technical considerations and longterm follow-up. BJU Int 2002;89:339-43. http://dx.doi.org/10.1046/i.1464-4096.2001.01562.x

5. Chaussy C, Brendel W, Schmiedt E. Extracorporeal induced destruction of kidney stones by shock waves. Lancet 1980;13:1265-8. http://dx.doi.org/10.1016/S0140-6736(80)92335-1

6. Chaussy $G C$, Fuchs $G J$. Current status and future developments of noninvasive treatment of urinary stones with extracorporeal shock waves lithotripsy. J Urol 1989;141:782-9.

7. Wickham JEA. The surgical treatment of renal lithiasis. In: Wickhamm JEA, editor. Urinary calculous disease. New York: Churchill Livingstone; 1979:183-6.

8. Raboy A, Ferzli GS, loffreda R, et al. Laparoscopic ureterolithotomy. Urology 1992;39:223-5. http:// dx.doi.org/10.1016/0090-4295(92)90294-7

9. Gaur DD. Retroperitoneal endoscopic ureterolithotomy: Our experience in 12 patients. J Endourol 1993;7:501-3. http://dx.doi.org/10.1089/end.1993.7.501

10. Lee WC, Hsieh HH. Retroperitoneoscopic ureterolithotomy for impacted stones. Changeng Yi Xue Za Zhi 2000;23:28-31.

11. Singh V, Sinha RJ, Gupta DK, et al. Transperitoneal versus retroperitoneal laparoscopic ureterolithotomy: A prospective randomized comparison study. J Urol 2013;189:940-5. http://dx.doi.org/10.1016/i. juro.2012.09.114

12. Sinha R, Sharma N. Retroperitoneal laparoscopic management of urolithiasis. J Laparoendosc Adv Surg Tech A 1997;7:95-8. http://dx.doi.org/10.1089/lap.1997.7.95

13. Lee $S Y$, Lee DH, Han WK, et al. Laparoscopic ureterolithotomy has a role for treating ureteral stones. Korean J Urol 2006;47:498-501. http://dx.doi.org/10.4111/kju.2006.47.5.498

14. Bozkurt OF, Resorlu B, Yildiz Y, et al. Retrograde intrarenal surgery versus percutaneous nephrolithotomy in the management of lower pole renal stones with a diameter of 15 to $20 \mathrm{~mm}$. J Endourol 2011;25:1131-5. http://dx.doi.org/10.1089/end.2010.0737

15. Resorlu B, Unsal A, Gulec $\mathrm{H}$, et al. A new scoring system for predicting stone-free rate after retrograde intrarenal surgery: The "resorlu-unsal stone score." Urology 2012;80:512-8. http://dx.doi.org/10.1016/i. urology.2012.02.072

16. Sun L, Peng FL. Treatment of ipsilateral renal ureteral calculi by combining retroperitoneal laparoscopic surgery with tubeless mini-percutaneous nephrolithotomy. Urol Int 2013;90:139-43. http://dx.doi. org/10.1159/000342104

Correspondence: Dr. Myung Ki Kim, Department of Urology, Chonbuk National University Medical School, 634-18, Geumam-dong, Dukijn-gu, Jeonju \#561-712, Korea; mkkim@jbnu.ac.kr 